\title{
Abnormalities in Plain X-Ray Findings of Lumbosacral Spine in Prolapsed Lumbar Intervertebral Disc.
}

\author{
Ansary $\mathrm{A}^{1}$, Mondle $\mathrm{M} \mathrm{S}^{2}$, Hossain $\mathrm{M} \mathrm{A}^{3}$
}

\begin{abstract}
:
This study was done prospectively over 50 admitted patients in the department of Neurosurgery, BSMMU from July'05 to June'06. Age range was 19-70 years, majority of them was male (76\%), 24\% was female, male: female was 3.17:1. Predominant occupation was manual labor (42\%), sedentary workers $24 \%$, housewives $26 \%$ and students $04 \%$. Out 50 patients $72 \%$ had right convexity and $28 \%$ had left convexity of the lumbosacral spine. Regarding lumbar lordosis $72 \%$ had straightened, 24\% had maintained and the remaining had reversed curve. Others $X$-ray findings were narrowing of interverteral disc spaces (76\%), osteophytes (52\%), narrowing of intervertebral foramina (46\%), sacralization (10\%), and listhesis $(10 \%)$. Out of that $57 \%$ had right sided prolapse and 38\% had left and 5\% had central.
\end{abstract}

The percentages of disc displace at one or two lowers grouped in the study as either a disc bulge is disc protrusion is $58 \%$. Disc bulge is more common than protrusion in this study with a prevalence of $42 \%$ compared to rest and the prevalence increases progressively caudally with the greatest number at L5/S1 level.

\section{Introduction:}

Low Back Pain is an increasing problem in the world. Most of the causes of low back pain is Prolapsed Lumbar Intervertebral Disc (PLI.D).

It occurs in high affluent societies, who are sedentary workers, as well as field workers (e.g. cultivators, rickshaw puller, mason, daily labour, etc.). Among low socioeconomic group, who are hard working people, lifting heavy weights in their daily life, doing strenuous work everyday.

PLI.D due to traumatic causes is mainly due to road traffic accident which is increasing day by day.

\footnotetext{
1. Dr. Ayub Ansari

Assistant professor, Department of Neurosurgery

Bangabandhu Sheikh Mujib Medical University,Dhaka

2. Dr. Md. Shamsuzzaman Mondle

Registrar, Department of Neurosurgery

Rajshahi Medical College Hospital, Rajshahi

3. Dr. .Mohammed Afzal Hossain

Professor and Head Department of Neurosurgery

Bangabandhu Sheikh Mujib Medical University,Dhaka
}

In the Affluent Society the cause of PLID with changes in lumbosacral spine is due to weak low back muscles for their minimum physical activities.

Most people suffer incapacitating Back Pain at some point in their lives. In fact, low back pain is second only to headache among the leading causes of pain ${ }^{1}$.

The lack of definitive diagnostic procedure in the past often made treatment of low back pain is frustrating and unrewarding, but today most patients with low back pain can return to active normal lives. Major advances made in our knowledge of the biomechanics of the spine and the mechanical causes of low back pain. However, our understanding of the nerve root entrapment syndrome is far from complete. The role of psychological factors is also recognized, but more research is needed on the complex interactions between the psyche and soma in the etiology of low back pain ${ }^{1}$

Bangladesh is a developing country. Most of our populations are in low socio-economic condition. In the Rural centers CT Scan or MRI. of the lumbosacral spine is not possible. In such conditions, basing on thorough Neurological Examination and plain x-ray of lumbosacral spine a clinical diagnosis can be established and further management can be planned. Either the patient can be operated on or if complicated can be referred to a tertiary hospital.

There are five lumbar vertebrae in human body, placed over the sacrum one above other with gradually increasing in size from above downwards. Facet joints are the main weight bearing joints in human body. The first four Lumbar vertebrae are typical and the fifth is atypical ${ }^{2}$.

The five lumbar vertebra in an articulated skeleton shows, articular facets lie in an anterior-posterior plane; they lock, and greatly limit rotation of the bodies on each other. Flexion and extension is free, and a good deal of abduction is possible; combinations of these movements produce circumduction ${ }^{3}$.

Abnormalities of lumbosacral spine are 3 types: 1) Congenital, 2) Degenerative, 3) Traumatic. They include -hemivertebra, sacralisation, lumbarisation, spina bifida, scoliosis, loss of lumbar lordotic curve, reverse lordotic curve, Reduction of intervertebral space, prolapse of intervertebral disc, compression fracture of vertebral body, 
burst fracture of vertebral body.

The spine as a whole has a static and dynamic functions. The lower lumbar Discs being submitted to more shock and strain than the others. The movement is maximal at the lumbosacral junction, explaining the high Incidence of disc disease at this level. Similarly torsion, strain, along with the burden of carrying weight result in frequent changes in the discs the mobility and curvature of lumbar spine varies with race. The African spine and the spine of Indians and Orientals are more mobile than those of Europeans, as the former squat on the floor and do most of their housework by stooping forwards ${ }^{4}$.

\section{Methods}

This cross sectional analytical and prospective study was done in the department of Neurosurgery Bangabandhu Sheikh Mujib Medical University among 50 patients from July'2005 to June'2006. The objective of this study along with risk and benefits of the study was explained to the patients or patient's attendant in easily under stable language and then a written informed consent was taken to each patient. The study was done with compatible history, plain x-ray of the lumbosacral spine AP and lateral view, myelogram of the lumbosacral spine, MRI of lumbosacral spine fulfills all inclusion criteria. Data was evaluated on the basis of detailed history, clinical examination, p-lain Xray and MRI of the lumbosacral spine. Those patients who improved with non-surgical treatment that is complete bed rest with analgesics or physiotherapy or consent to surgery denied by the patient or his guardian or age more than 70 years were excluded. A checklist was prepared by the researchers considering the variables such as age, sex of the patients, clinical presentations, straight leg raising test, radiculopathy and bowel bladder involvement.

Imaging variables were measured by MRI scan, myelogram, plain X-ray of lumbosacral spine. Variables studied were -1) Space of disc prolapse, 2) in which side the prolapse of disc occurred, 3) which never root was involved, 4) disc was protruded or extruded, 5) there was scoliosis or loss of lumbar lordotic curve.

The checklist was tested first in the department of Neurosurgery, BSMMU. Then finalized the data as collected by the researchers themselves by standard technique. Data was evaluated by the findings of MRI scan or myelogram and plain x-ray with standard biostatical programme and if required resource personal in the field of biostatistics will be consulted.

\section{Result}

A total of 50 patients of low-back pain were selected to find the changes in plain X-ray findings of lumbosacral region. The findings of the study obtained from data analysis are presented below (Table-1).

\begin{tabular}{|l|l|c|}
\hline Age (yrs)\# & No & \% \\
\hline$<20$ & 01 & 2.0 \\
\hline $20-30$ & 06 & 12.0 \\
\hline $30-40$ & 21 & 42.0 \\
\hline $40-50$ & 11 & 22.0 \\
\hline$\cdot 50$ & 11 & 22.0 \\
\hline
\end{tabular}

Table-I: Age distribution of the patients $(n=50)$ :

\# Mean age $=(38.62 \pm 10.15)$ years; range $=(19-70)$ years. Table shows that highest frequency of age (42\%) ranged $30-40$ years.

\section{Fig. 1: Sex distribution of the patients}

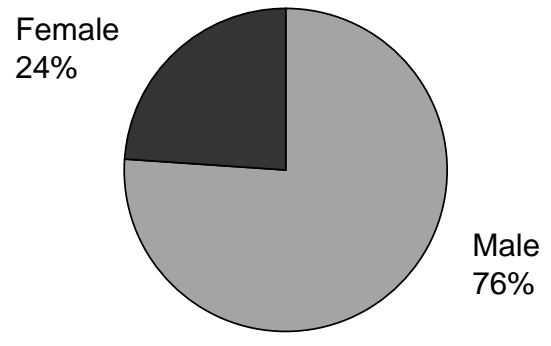

Fig-I shows that over three-quarters $(76 \%)$ patients were male and $24 \%$ were female giving a male-female ratio of $3.17: 1$.

\begin{tabular}{|l|c|c|}
\hline Occupation & No & \% \\
\hline Manual labour & 21 & 42.0 \\
\hline Housewife & 13 & 26.0 \\
\hline Sedentary worker & 12 & 24.0 \\
\hline Student & 02 & 4.0 \\
\hline Others & 02 & 4.0 \\
\hline
\end{tabular}

Table-II: Distribution of the patients by occupation $(n=50)$ :

Table-II demonstrates that predominant occupation of the patients was manual labour $42 \%$. Other occupants were housewives $(26 \%)$ sedentary workers $(24 \%)$, students $4 \%$ and other $4 \%$ military and paramilitary workers.

regarding precititating factors $40 \%$ by sneezing, $32 \%$ by straining, $30 \%$ by weight lifting and $10 \%$ by trauma. RTA the patients informed that their pains were aggravated by movement, $44 \%$ by coughing, and multiparity each was complained by $1(2 \%)$ patient.

Table: Distribution of patients by relieving factors $(n=50)$ :

Out of 50 patients $49(98 \%)$ told that pain was relieved by taking rest by lying down and/or by taking analgesics.

$12 \%$ of the patients complained of radiation of pain down to calf muscle, $50 \%$ down to heel and $38 \%$ even down to toes.

Straight leg raising test of right leg $40 \%$ of the patients were able to raise their leg at 900 angle followed by $14 \%-750$, 
$18 \%-600,20 \%-450,16 \%-300$ and $2 \%-150$.

While on straight leg raising test of left leg $30 \%$ of the patients were able to raise their leg at 900 angles followed by $14 \%-750,18 \%-600,20 \%-450,16 \%-300$ and $2 \%-150$.

The study depicts that majority (92\%) of the patients did not have any problem of bowel-bladder habit and the rest $8 \%$ had such problem. The study shows that $94 \%$ of the patients did not exhibit saddle anesthesia, while the rest $6 \%$ exhibited such problem. Study presents that more than half $(54 \%)$ of the patients did not have any sensory changes, while $38 \%$ had hypoesthesia and $8 \%$ had hyperesthesia.

In the study of muscle power of the patient, $70 \%$ of the patients did not demonstrate any abnormality in muscle power. The rest $30 \%$ showed diminished muscle power.

More than $80 \%$ of the patients normally extended their left toes, while $18 \%$ exhibited weak extension. Forty six percent of the right toes were normal and 54\% were weak in terms toe extension.

More than three-quarters (78\%) of right knees and $90 \%$ of the left knees exhibited normal jerks. Fourteen percent of the right knee jerks were diminished, $6 \%$ absent and $2 \%$ increased. Of the $10 \%$ abnormal left knee jerks, $6 \%$ were diminished and $4 \%$ absent.

$86 \%$ of the left ankles and $54 \%$ of the right ankles retained normal jerks. Nearly one-quarter (24\%) of the right ankle jerks were diminished, $20 \%$ absent, and only $2 \%$ increased. Of the $14 \%$ abnormal left ankle jerks, $8 \%$ were diminished and $6 \%$ absent.

\begin{tabular}{|l|c|c|}
\hline Scoliosis & No & \% \\
\hline Right convexity & 36 & 72.0 \\
\hline Left convexity & 14 & 28.0 \\
\hline
\end{tabular}

Table-III: Distribution of patients by scoliosis $(\mathrm{n}=50)$ :

Table-III depicts out of 50 patients 36 (72\%) of the patients had right convexity, while $14(28 \%)$ patients demonstrated left convexity of vertebral column.

\begin{tabular}{|l|c|c|}
\hline Lumbar lordosis & No & \% \\
\hline Maintained & 12 & 24.0 \\
\hline Straightened & 36 & 72.0 \\
\hline Reversed & 2 & 4.0 \\
\hline
\end{tabular}

Table-IV: Distribution of patients by lumber lordosis $(\mathrm{n}=$ 50):

Table-IV describes that out of 50 patients $12(24 \%)$ patients maintained lumber lordosis, $36(72 \%)$ straightened and $4 \%$ reversed.

\begin{tabular}{|l|c|c|}
\hline Other X-ray findings & No & $\%$ \\
\hline Sacralisation & 05 & 10.0 \\
\hline Listhesis & 03 & 6.0 \\
\hline Narrowing of intervertibral space & 38 & 76.0 \\
\hline Osteophytes & 26 & 52.0 \\
\hline Narrowing of intervertibral foramina & 23 & 46.0 \\
\hline
\end{tabular}

Table-V: Distribution of patients by other X-ray findings $(\mathrm{n}=$ $50 *)$ :

*Total will not correspond to $100 \%$, because of combined occurrence of $>1$ features in some patients.

Table-V demonstrates the other X-ray findings. Of the other $\mathrm{X}$-ray findings more than three-quarters $(76 \%)$ had narrowing of intervertebral space, $52 \%$ osteophytes, $46 \%$ narrowing of intervertebral foramina, $10 \%$ sacralisation and the rest $6 \%$ had listhesis.

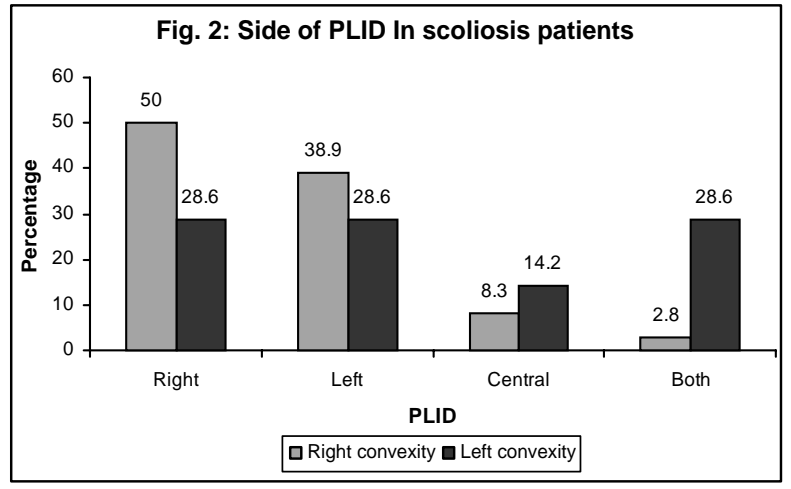

Fig-2. demonstrates that $50 \%$ of the patients of scoliosis with right convexity had right-sided PLID followed by $38.9 \%$ left-sided PLID, $8.3 \%$ central and $2.8 \%$ both right and left sided PLID. On the other hand, $28.6 \%$ patients of scoliosis with left convexity had right PLID, 28.6\% left PLID and another $28.6 \%$ had both right and left. While $14.2 \%$ had central PLID.

Nearly half $(47.2 \%)$ of the scoliosis with right convexity exhibited PLID at the level of L4 - L5, 38.9\% at L5 - S1, $8.3 \%$ at $\mathrm{L} 4-\mathrm{S} 1$ and $5.6 \%$ at L3 - L4. More than one-third $(35.7 \%)$ of the scoliosis with left convexity demonstrated PLID at L4 - L5 followed by $21.4 \%$ at L5 - S1, another $21.4 \%$ at $\mathrm{L} 4-\mathrm{S} 1,14.3 \%$ at L3 - L4 and only $7.1 \%$ at L2 L3.

Table: Side of PLID in patients with sacralisation $(n=5)$

Out of 5 patients with sacralisation $3(60 \%)$ had right-sided PLID, 1(20\%) had left-sided PLID and the rest 1(20\%) had both sided PLID.

Out of 3 patients with listhesis $1(33.3 \%)$ had right-sided, $1(33.3 \%)$ had left-sided and 1(33.3\%) had both sided PLID. 
Out of 3 patients with listhesis $2(66.7 \%)$ had PLID at L4 L5, and 1(33.3)\% at L3 - L4.

Out of 38 patients with decreased intervertebral space $17(44.7 \%)$ had right-sided, $13(34.2 \%)$ had left-sided, $5(13.2 \%)$ had both right and left sided and $3(7.9 \%)$ had central PLID.

Table depicts that out of 38 patients with decreased intervertebral space nearly $40 \%$ had PLID at L4 - L5, followed by $14(36.8 \%)$ at L5 - S1, 3(7.9\%) at L3 - L4 and $1(2.6 \%)$ at L2 - L3. Five patients (13.2\%) had PLID at L4 $\mathrm{L} 5$ as well as at L5 - S1.

\section{Discussion}

In this study, total of 50 patients with low back pain due to PLID was selected to find out the changes in plain $x$-ray findings of the lumbosacral spine. The findings of the study obtained from data analysis are presented below.

Age ranged 19-70 years with highest frequency was at the age group of 30-40 years

In United States, the most commonly affected age group is 25-45 years 5 . Ming from TCM Medical center, China, states that prolapsed lumbar intervertebral disc occurred often in youth and middle aged of 20-40 [6]. Regarding sex, over majority $(76 \%)$ patient was male and $24 \%$ was female, giving a male - female ratio of 3.17:1. In our neurosurgery department, as like other special subjects, bed numbers of male patients are 3 times greater than that of female. So, male has better chance of admission and getting more chances of investigations and treatments. But in United States and other developed countries the male-to-female ratio is approximately $1: 15$. . Dr. Ming from TCM Medical Centre, China, states that in PLID males surpasses the female in number ${ }^{6}$.

Regarding occupation, predominant occupations of the patients were manual labor $(42 \%)$ and sedentary worker (24\%). Other occupants were housewives (26\%) and athletes $(04 \%)$ Because of the physical labor particularly apparent in rural and isolated human populations, in which agriculture and fishery represent the main human activities. Because the physical labor they perform requires working most of the time in bending position that particularly burdens the lower spine, they have increased lifetime risk of lumbar disc herniation. In a sample of 1001 examinees Croatian island populations, where they identified all subjects who underwent surgery of the lower spine due to lumbar disc herniation L/4L5 or L5/S1.Comparision of 67 identified cases with 268 controls revealed the intensity of physical labour at work defined as "hard" (OR 2.77, 95\%)8. Compared with changes seen in normal population (nonathletes) disc degeneration defined to be significantly more severe in Olympic athletes who had disc height reduction at a single level is $68 \%$. Because of the human sedentary nonmoving lifestyle, disc degeneration is progressive.
Regarding precipitating factors that induce pain in PLID cases, $30 \%$ of the patients informed that their pains were aggravated by weight lifting, $10 \%$ following a trauma, $44 \%$ by coughing and $40 \%$ by sneezing.

Out of 50 patients , 98\% told that pain was relieved by taking rest by lying down and in remaining pain was relieved by rest by sitting or standing (Some patients have more than one relieving factors). Symptoms are mitigated by lying down on one side with hip and knee flexed (fetal position) and changing position, observed by Patel in 2006 .

Almost half (49\%) of the PLID patients had right side involvement, and one third (32\%) had left side involvement and $(19 \%)$ had both side involvements.

Out of 50 patients $72 \%$ had right convexity scoliosis and $28 \%$ left convexity scoliosis in the lumbosacral spine. Matsui et al. observed that $80 \%$ of patients with disc herniation and scoliosis had a convexity on the side of the root compression ${ }^{10}$.

The chronic spasm of low back muscles causes straightening of lumbar lordotic curve. In this study out of 50 patients, $72 \%$ had straightening, $24 \%$ maintained lumbar lordosis, and $4 \%$ reversed curve. Hyperlordosis occurs due to wearing high heels (they push the body forward and force a hyperlordotic attitude to rebalance it. It also occurred in pregnancy and sleeping with belly downwards on a soft mattress ${ }^{11}$.

Of the other plain $\mathrm{x}$-ray findings of lumbosacral spine, $10 \%$ had sacralisation, $76 \%$ had narrowing of inververtebral space, listhesis $06 \%$, and osteophytes in $05 \%$ cases.

Present study showed that there is a correlation between the changes which occurs in lumboscaral spine in PLID cases.

Proper care of low back muscles by doing regular specific exercises of low back muscles which strengthens the muscles of low back and prevents further changes in the lumbosacral spine (e.g. Scoliosis and straightening of lumbar lordotic curve).

To prevent changes in the lumbosacral spine, patient should be advised to use commode in the toilet and to avoid pan to protect low back muscle from straining or pressure on it.

Patient with low back pain should be advised not to lift any heavy weight and to avoid giving pressure in low back muscle by any means. Proper training for getting up carefully from lying or sitting positions should be advised.

The patient who developed progressive neurological deficit, saddle anaesthesia or progressive neurogenic claudication should be operated urgently for better and reversible out come.

Degeneration of the intervertebal disc begins early in life and is partly a consequence of aging. Powel et al observed 
that more than one third of normal healthily subjects aged 21-30 years had degenerated discs. Although the actual cause is not known, many factor (autoimmune, genetic, reabsorption, and biomechanical) have been implicated in accelerating process. The volume of the intervertebral disc tissue decreases with degeneration, and it has been shown that failure of the human lumbar intervertebral disc occurs most often in the part of the spine that is subjected to have heaviest mechanical stress. The results of several studies have shown that the level of physical activity affects disc height. However, Luoma et al have questioned the validity of disc height as an indicator of early disc degeneration. In the study, the proportion of discs with reduced height gradually increased in a caudal direction, with the greatest prevalence at the L5/S1 level. (Ong, 2003)

Similarly, signal of the lumbar intervertebral discs becomes progressively reduced caudally. This trend is similar to the results obtained by De Candido et al. In their study, at the L1/2 level, $81 \%$ of discs have normal signal intensity (compared with $78 \%$ found by DeCandido et al), which then progressively decreases to $38 \%$ (40\% found by DeCandido et al) at the L5/S1 level (Ong, 2003).

\section{Summary}

Abnormalities in plain x-ray findings in PLID cases mainly occurs in 1) Congenitally abnormal spine of 2) The changes in the lumbosacral spine, which occurs in chronic low back pain due to never root compression or irritation in PLID cases. The continuous never root compression or irritation causes para spinal muscle spasm and this continuous spasm is the main cause of the scoliosis of the lumbosacral spine, found in the AP view of plain x-ray of the lumbosacral spine. The convextty of scoliosis of the lumbosacral spine is opposite to the never root compression, which gives temporary relief to the patient by the release of entrapped never root, where the disc space becomes reduced in PLID cases.

In the lateral view of plain x-ray of lumbosacral spine, the changes are found as - straightening of lumbosacral spine, which usually occurs in most of the PLID cases due to chronic para spinal muscle spasm. In long standing cases with severe para spinal muscle spasm, the lumbar lordotic curve may be reversed.

These changes in lumbosacral spine (Scoliosis, straighteing of the lumbar lordotic curve or reversal of the lumbar lordotic curve) improve slightly or not at all with conservative treatment or of surgical decompression (fenestration and discectomy of the spinal deformity). But the treatment stops further progression of the spinal deformity. When the deformity becomes excessive, internal fixation by screw-plating may be required to protect further deformity.
In acute cases, most of the changes in the lumbosacral spine occur due to trauma.

The operations mainly performed in PLID cases are fenestration and discectomy, microdiscectomy and laminectomy. Microdiscectomy or fenestration and discectomy are preferred over laminectomy because laminectomy weakens the spine and may cause further deformities in lumbosacral spine. But microdiscectomy of fenestration and discectomy stops further progression of deformity of lumbosacral spine such as scoliosis or straightening of lumbosacral spine.

In case of formation of facet or narrowing intervertebral foramen, facetectomy or foraminotomy gives very good results.

\section{Conclusion}

There are significant changes found in the plain x-ray findings in most of the cases of PLID. The changes found in plain x-rays are mostly scoliosis, loss of lumbar lordotic curve (These are mostly due to chronic spasm of the low back muscle due to chronic irritation of nerve roots by the disc or osteophytes). Other changes found in plain x-ray lumbosacral spines are: sacralisation, reduction of intervertebral space, spina bi-fida(congenital, osteophytes causing narrowing of intervertebral foramina. Ossified posterior longitudinal ligament, Hypertrophied or thickened ligamentum flava may cause canal stenosis causing neurogenic claudication. Hypertrophy of the facet joint causes compression on nerve roots which causes radulopathy.These bony abnormalities acts as predictor for future possibility of PLID.

\section{References:}

1. Keim HA, Kirkaldy WWH ,1987, Clinical Symposia on low back pain.

2. ChaurasiaHuman Anatomy, Regional \& Applied, Inderbir Singh, CBS Publishers \& Distributor, Delhi, 1991, 2: 151-158.

3. Mc Minn RMH, Last's Anatomy. Regional \& Applied, Churchill. Livingstone, Medical Division of Longman Group Ltd, UK, 8th ed, 1993, 40-543.

4. Goel A., Pandya SK, Lumbar Disc Protrusion, in Ramamurthi, Tandon PN, Text Book of Neurosurgery, 2nd ed, BI Churchill Livingstone Pvt; New Delhi, 1996, 2:743-756.

5. Baldwin J, Lumbar Intervertebral Disc Disorders,Mark S. Slabinski et all., Southern

Ohio Regional Center, e medicine, 2006, 2:1-11.

6. Ming, Yuan HXX., Prolapse of Lumbar Intervertebral disc, Dr. Mings Medical center China, 2001, 1:1-2 
7. Greenberg MS, Handbook of Neurosurgery, 4th ed, Greenberg Graphics, Florida, 1997, 2:285-

300 .

8. Staftic R., Grgic M., Ebling B, Intervertebral Disc Herniation in Croatian Island

Population, Robert Staft ,Case Control study of Risk factors for ic, 31000 Osijec, Croar Med J. July 10, 2006 Croatia; 47:593-600.

9. Ong, Anderson J. Roche J, A Pilot Games, study of the prevalence of Lumbar disc degeneration in elite athlets with lower back pain at the Sydney 2000 Olympic, Dr. Ong, Sydney, Australia, 2003, 37: 263-266.

10. Pinto FCG, Poetscher AW, Quinhones FRE, Penna M, Toricco MA, Lumbar Disc Herniation Associated with Scoliosis, Sao Paulo, Sei Elo, Brazil, 60:2A.

11. Keisermam M., 2002, Lumbar Pain, Brazillian Society of Rheumatology, 2002, 1-7. 